TECHNICAL NOTES AND SHORT PAPERS

\title{
The Approximate Solution of an Integral Equation Using High-Order Gaussian Quadrature Formulas
}

\author{
By Stephen M. Robinson and A. H. Stroud
}

The purpose of this note is to illustrate the usefulness of the high-order Gaussian quadrature formulas given in [1], [2], [3] in a problem of approximating the value of

$$
I=\int_{a}^{b} g(x) \varphi(x) d x
$$

where $\varphi(x)$ is the solution of a Fredholm integral equation

$$
\varphi(x)-\int_{a}^{b} K(x, y) \varphi(y) d y=f(x)
$$

Here $K(x, y), f(x)$ and $g(x)$ are known functions and $K(x, y)$ has a discontinuity at $y=x$.

The approximate solution of (2) was found by a method described in [4], p. 101. It consists of replacing (2) by

$$
\varphi(x)\left[1-\int_{a}^{b} K(x, y) d y\right]-\int_{a}^{b} K(x, y)[\varphi(y)-\varphi(x)] d y=f(x)
$$

Now, since $K(x, y)$ is a known function, $\int_{a}^{b} K(x, y) d y$ can be determined as a function of $x$, say $\chi(x)$. It is reasonable to suppose that the integral

$$
\int_{a}^{b} K(x, y)[\varphi(y)-\varphi(x)] d y
$$

can be better approximated by a quadrature formula than the original integral in (2). If we use a quadrature formula with weights $A_{k}$ and points $x_{k}, k=1,2, \cdots, n$, to evaluate (3), then approximate values of the function $\varphi\left(x_{k}\right)$ may be determined by solving the linear system

$$
\varphi\left(x_{i}\right)\left[1-\chi\left(x_{i}\right)\right]-\sum_{k=1}^{n} A_{k} K\left(x_{i}, x_{k}\right)\left[\varphi\left(x_{k}\right)-\varphi\left(x_{i}\right)\right]=f\left(x_{i}\right) \quad i=1,2, \cdots, n .
$$

The same quadrature formula may then be applied to approximate the integral (1).

To illustrate the accuracy of Gaussian quadrature formulas in this problem we give the results of some calculations concerning flow of a gas through a conical opening which were carried out for Prof. R. P. Iczkowski of the University of Wisconsin Extension Chemistry Department. The kernel $K(x, y)$ was defined for two

Received December 29, 1960. The preparation of this note was supported in part by the Graduate Research Committee of the University of Wisconsin. 
TABLE 1

Values of $I$

\begin{tabular}{c|c|c|c}
\hline$n$ & $\begin{array}{c}\alpha=0 \\
L=2\end{array}$ & $\begin{array}{c}\alpha=10 \\
L=2\end{array}$ & $\begin{array}{c}\alpha=10 \\
L=10\end{array}$ \\
\hline 12 & 0.5142287 & - & - \\
16 & 0.5142294 & - & - \\
24 & 0.5142305 & 0.6705783 & 0.4768471 \\
36 & 0.5142306 & 0.6706079 & 0.4775303 \\
48 & 0.5142306 & 0.6706184 & 0.4777735 \\
\hline
\end{tabular}

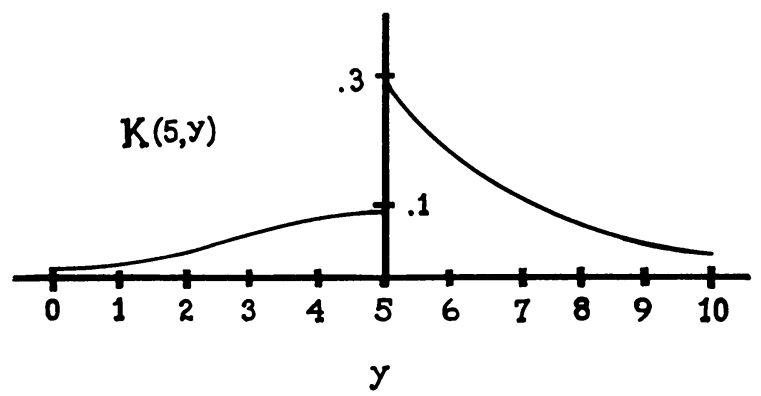

FIG. 1.-Cross-Section of the Kernel $\mathrm{K}(\mathrm{x}, \mathrm{y})$.

parameters, $\alpha$ and $L$. The table gives values of $I$ for three cases; in each case $I$ was evaluated using the indicated $n$-point Gaussian quadrature formulas. The calculations were carried out using single-precision, floating point arithmetic.

The nature of the kernel is illustrated in the figure which gives a typical crosssection of $K(x, y)$.

The calculations for $\alpha=0, L=2$ are a good illustration of the accuracy achieved over most of the range investigated: $\alpha=0$ to $89, L=0.1$ to 10.0 . In this case the 24-point formula seems to give 6-decimal accuracy. The other cases of the table are some of the least accurate. For, $\alpha=10, L=10$ the 24-point formula seems to give 3-decimal accuracy and the 48-point formula about 4-decimal accuracy. In all of these calculations it appears that, roughly, doubling the number of points produces another decimal place of accuracy. No analytical estimates of the error were carried out. These calculations illustrate, however, that by using 8 significant figures in such calculations it is possible to achieve 6- or 7-figure accuracy in the result if a sufficiently accurate formula is used.

The calculations for $n=12,16$ were carried out on the IBM 650 at the Numerical Analysis Laboratory and for $n=24,36,48$ on the IBM 704 at the Midwestern Universities Research Association. The programs were written by Mr. Robinson. Funds for the computations were provided by the University of Wisconsin Chemistry Department.

Numerical Analysis Laboratory

University of Wisconsin

Madison, Wisconsin

1. P. Davis, \& P. RaBinowitz, "Abscissas and weights for Gaussian quadratures of high order," J. Res. Nat. Bur. Standards, v. 56, 1956, p. 35-37. 
2. P. Davis, \& P. Rabinowitz, "Additional abscissas and weights for Gaussian quadrature of high order: values for $n=64,80$, and 96," J. Res. Nat. Bur. Standards, v. 60, 1958, p. $613-614$.

3. H. J. GAwLIK, "Zeros of Legendre polynomials of orders 2-64 and weight coefficients of Gauss quadrature formulae," Armament Research and Development Establishment Memoran$\operatorname{dum}(B) 7 \gamma / 58$, Fort Halstead, Kent, 25 p., Dec. 1958.

4. L. V. Kantorovich, \& V. I. KRYLOv, Approximate Methods of Higher Analysis, Interscience \& Noordhoff, New York and Groningen, 1958.

\section{Bi-Variate, Rectangular, Optimum-Interval Interpolation}

\section{By Ferdinand Freudenstein}

1. Definition of "Optimum-Interval" Interpolation. Let the real function $f(x, y)$, defined in the interval $-h \leqq x \leqq h,-k \leqq y \leqq k, h>0, k>0$, and assumed to have continuous partial derivatives of order up to $(m+n+2)$ in the rectangle, be approximated by the polynomial

$$
P_{m n}(x, y)=\sum_{r=0}^{m} \sum_{8=0}^{n} a_{r s} x^{r} y^{8}
$$

so that at the $(m+1)(n+1)$ precision points, $\left(x_{u}, y_{v}\right), u=0,1,2, \cdots m$; $v=0,1,2, \cdots n$,

$$
P_{m n}\left(x_{u}, y_{v}\right)=f\left(x_{u}, y_{v}\right)
$$

In that case,

$$
f(x, y)=P_{m n}(x, y)+R_{m n}
$$

where the remainder, $R_{m n}$, is given by the expression (see [1])

$$
\begin{array}{r}
R_{m n}=\frac{1}{(m+1) !} \prod_{u=0}^{m}\left(x-x_{u}\right) \frac{\partial^{m+1} f(\xi, y)}{\partial \xi^{m+1}}+\frac{1}{(n+1) !} \prod_{v=0}^{n}\left(y-y_{v}\right) \frac{\partial^{n+1} f(x, \eta)}{\partial \eta^{n+1}} \\
-\frac{1}{(m+1) !(n+1) !} \prod_{u=0}^{m}\left(x-x_{u}\right) \prod_{v=0}^{n}\left(y-y_{v}\right) \frac{\partial^{(m+1)+(n+1)} f\left(\xi^{\prime} \eta^{\prime}\right)}{\partial \xi^{\prime m+1} \partial \eta^{\prime n+1}}
\end{array}
$$

where $\xi, \xi^{\prime}\left(\eta, \eta^{\prime}\right)$ lie between the greatest and least of the numbers $x, x_{u}\left(y, y_{v}\right)$.

Consider the set, $S$, of all precision points $\left(x_{u}, y_{v}\right)$ within the rectangle $(-h, h)$ $(-k, k)$-called $(h, k)$ from now on. $S$ will be called the spacing. Let $h=k \delta$, where $\delta$ is a positive number defining the proportions of the rectangle. Furthermore, we restrict ourselves to functions for which the partial derivatives $\frac{\partial^{m+1} f(x, y)}{\partial x^{m+1}}, \frac{\partial^{n+1} f(x, y)}{\partial y^{n+1}}$, $\frac{\partial^{(m+1)+(n+1)} f(x, y)}{\partial x^{m+1} \partial y^{n+1}}$ are not equal to zero within $(h, k)$. Let $R$ be the largest lower limit on the maximum absolute value of $R_{m n}$ regardless of spacing. It is assumed

Received September 5, 1958; final revision April 22, 1960. 